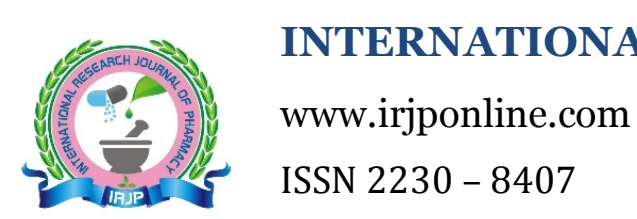

\title{
Research Article \\ ORAL CANCER PREVALENCE \& FINDING OF ALARMING CONSEQUENCES AT ONCOLOGY WARD OF PUBLIC HEALTH CARE SECTOR
}

Maria Ayub *, Mehreen fayyaz, Sumra arshad, Bushra riaz khan, Uzma bano, Falak khalil

Jinnah University for women, Karachi 74600, Pakistan

*Corresponding Author Email: maria.ayub2000@gmail.com

Article Received on: 25/06/15 Revised on: 27/07/15 Approved for publication: 19/08/15

DOI: $10.7897 / 2230-8407.069121$

\begin{abstract}
Oral cancer is one of the most occurring cancers from 1908.Its prevalence is rapidly increasing day by day and the most commonly occurring type is squamous cell carcinoma from which more than 50,000 patients affected per year around the world. In this study we are going to describe the main consequences of oral cancer along with its prevalence \& consequences responsible for oral cancer. This study deals with the some of the main consequences of oral cancer with their percentage of causing oral cancer including tobacco intake,alcohol consumption,smoking, genetically inherited factor,effect of sunlight and use of red chili in higher amount.For the purpose of searching its main consequences the efforts were made which are based on the survey of different hospitals and reading out the case history and profile of more than 100 patients. This survey was conducted in different hospitals, diagnostic and treatment centers of Karachi and as a result of this survey the collective efforts that achieved, revealed that those patients who were habitual of taking tobacco are at higher risk (of about $50 \%$ ) of developing oral cancer. Then the $2^{\text {nd }}$ most common factor involved in oral cancer is alcohol consumption of about $30 \%$. Along with these two, several factors are also involved including genetic factor, smoking, sunlight and many more. On the basis of this survey it is concluded that the above mentioned consequences are greatly responsible for oral cancer \& its prevalence can be prevented by get rid of the main consequences of oral cancer mentioned in this study.
\end{abstract}

Keywords: Oral cancer consequences, oral cancer prevalence, oral cancer and HPV

\section{INTRODUCTION}

Oral cancer is consider to be the sixth most common cancer all over the world, but its prevalence is high found in south Asian ${ }^{1}$.Oral cancer is most common in south central Asian men than women ${ }^{2}$.In Pakistan the incidence of oral cancer and oropharyngeal cancer are very high ${ }^{3}$.Cancer of oral cavity and pharynx are among the most common type of cancer according to the report which is published by Pakistan Medical Research Council (PMRC) $)^{4,5,6}$.

Oral cancer is generally known as mouth cancer, Over $90 \%$ arises from the oral mucosal lining tumors of squamous cell carcinoma ${ }^{7}$ .Squamous cell carcinoma is the most common form of oral cancer, originated in mucosal epithelium, occur more frequently with pain $^{8}$.Oral cancer is the neoplasm of head and neck great morbidity cause by this disease, so many approaches has been done to prevent the cancer the most common is prevent oral lesion or detection in patient with oral leukoplakia. Oral leukoplakia is the white patch in the oral cavity they may exceed the risk of the cancer, leukoplakia is considered as the marker of cancer9.

Early sign and symtoms of oral cancer can be seen are sudden tooth mobility without any cause, a lump and a lateral lump in the neck, mouth sore which is fail to heal early or unusual bleeding ${ }^{10}$. The typical sign and symptoms of oral cancer are sore throat or mouth over three weeks a swelling in the mouth that persist mouth ulcers, pain during swallowing, without any reason loosening teeth, pain in jaw with jaw stiffness, thickening or lump in the oral cavity, hoarse voice, painful tongue ${ }^{11}$.

Major risk factors of oral cancer which have been seen are chewing tobacco, smoking, chewing of beter quid, the excessive consumption of alcohal, body being exposed to sunlight for long period of time (associated with lip cancer), nutrition and immune deficiencies are most emerging risk factor of oral cancer ${ }^{12}$.In the formation of mouth cancer the main etiological factor is HPV Humanpapillomavirus that be analyzed by using some experimental methods ${ }^{13}$.

Some studies shows that the over expression of COX-2 enzyme is involved in many cancers specially the cancer of upper digestive tract like oral cancer ${ }^{14}$.by promoting growth of tumors, inducing angiogenesis, by stopping apoptosis and by invasion and metastasis their incidences show that COX-2 induces carcinogenesis and these all are considered to be the main hall mark of mouth cancer. 1195A > $\mathrm{G}$ has no effect, whereas COX-2 polymorphism-765 G $>\mathrm{C} \&+837 \mathrm{~T}$ $>\mathrm{C}$ are at high risk of oral cancer ${ }^{15}$.

Nitric oxide is a gas and also serves as a neurotransmitter and vasodilator ${ }^{16}$.iNOS over expression has also been observed in squamous cell carcinoma ${ }^{17}$.In the progression of squamous cell carcinomas P53 and iNOS expression is strongly co-related ${ }^{18}$.

Head and neck cancer also cause by infection of HPV human papillomavirus ${ }^{19}$.HPV is said to be responsible to immobilize the epithelial cells and if it is used with tobacco and alcohol and others chemicals it lead to synergistics effect and this effect is the key that induces carcinogenesis which is link to $\mathrm{HPV}^{20}$.Carcinoma of squamous cell is also associated with the presence of visile bacteria within the oral cavity by using standar microbiological culture approach thus bacteria primary can be identified with oral squamous cell. These bacteria includes, Acientobacter lwoffi, Olsenella uli, Lactobacillus crisputus, Canadida albicans ${ }^{21}$.

Some diseases which are also responsible for oral cancer such as oral sub mucous fibrosis (OSF) a disease of oral cavity and mucous of oral cavity having the characteristics of inflammation anf fibrosis of lamina propria and some on its transformation to malignancy, the 
main etiological factor in the formation of mouth cancer is oncogenic connective tissues in depth ${ }^{22}$.

The genetic factor is also involved in oral cancer. A study shows that about $91.67 \%$ of genes and about $37.5 \%$ of miRNAs are involved in oral carcinomas, about $2-3 \%$ cases of leukoplakia are said to be involved in oral cancer annually. And this transformation of leukoplakias to carcinomas involves 4 sub genes that are, STAT5B, EGFR, PDGFRB, STAT5A. And 5 inter-MAOC genes involved are PRKCA, MAPK1, MAPK3, ESR1, and FYN. miRNAs involved are of 8 kinds including, hsa-miR-499-5p, hsa-miR-549, hsa-miR-5255p, hsa-miR-21, hsa-miR-3323-3p, hsa-miR-423p, hsa-miR-3p ${ }^{23}$.

Some studies have evaluated that a tumor suppressor gene WWOX gene which is responsible for molecular changes related to oral cancer its location is 16q23.3-24.1 and it spans FRA16D found in many malignant neoplasia which is the secondary most common fragile site, in which oral squamous cell carcinoma is also included ${ }^{24}$.In a case control study it has been observed that many factors are involved in oral carcinomas but the ratio of drinking and smoking has the highest risk of developing $\mathrm{it}^{25}$.environmental pollutant also proved to be contributing factor in other type of cancer ${ }^{26}$.various studies conducted at oncology section clearly reflects lack in awareness about cancer causing agents especially in breast carcinoma patients ${ }^{27}$.

In this study we aimed to findout the prevalence of oral cancer and consequences responsible against this life threatening cancer which create alarming situations at Pakistan society in view of the fact to encounter such type of big evil in public healthcare sectors.

\section{METHODOLOGY}

This study was conducted in 2015 in Karachi within one month which is based on the data obtained by the survey of different oncology departments of different hospitals and by viewing of case histories and profiles of more than 100 patients of oral and its related cancers. And in this the patients were directly interviewed about their case. This research involves the out patients (on the day of OPD) as well as IPD. Different hospitals were visited many times in order to collect complete and proper information. Mostly the patients with oral cancer were found on the day of OPD because most of the patients of oral cancer are not admitted in the hospitals unless it is severe form of cancer or any surgery would have to be done for that severe form. Most of the patients are found with the consequence of using tobacco about $50 \%$ of the total patients surveyed. Other consequence of oral cancer found in patients is alcoholism up to $30 \%$, smoking about $10 \%$, sunlight up to $2 \%$, use of high amount of red chili up to $3 \%$. The factor of inheritance was also found in some patients up to $4.5 \%$. The consequence of oral cancer in individual with A-blood group can also be seen in some patient registries but to a lesser extent of about $0.5 \%$.Oral cancer types found in patients are squamous cell carcinoma most commonly, tongue cancer, lips cancer and throat cancer that are associated with mouth cancer.

\section{RESULT}

For this survey many patients' profile were observed carefully and on basis of these finding it can be said that oral cancer is linked to a number of consequences and all these consequences are in turn linked to the development of many kinds of oral carcinomas as shown in Table.1. And to decrease the prevalence of oral cancer, withdrawal of tobacco is must as it is at high rate of developing oral cancer of any type. Table. 1 shows the types oral cancer with respect to their consequences. And all of the consequences have their own mechanism in the formation of specific type of mouth cancer. But some consequences have the ability to develop any kind of carcinoma which is at the greatest risk of developing oral cancers.
Table 1: common type of cancer

\begin{tabular}{|c|c|}
\hline CONSEQUENCES & COMMONLY CAUSED TYPE \\
\hline Tobacco & Of Any Type \\
\hline Alcohol & Usually Throat Cancer \\
\hline Smoking & Mouth, Nose and Throat Cancer \\
\hline Genetic Factor & Any Type \\
\hline Sunlight & Lip Cancer ${ }^{17}$ \\
\hline Red Chili Use & Throat And Mouth cancer \\
\hline Viral Infections & Oropharynx Cancer ${ }^{17}$ \\
\hline
\end{tabular}

\section{Statistical Analysis}

The statistical analysis for the consequences of oral cancer in Fig.1 shows that tobacco is the $1^{\text {st }}$ main cause of oral cancer that is largely involved in different types of mouth carcinomas. And the $2^{\text {nd }}$ main consequence is alcohol consumption. These two consequences when combined means if both of these are used by a single person is said to be at the greatest risk of developing oral carcinoma than that of a person who consume any one of them. Smoking is the $3^{\text {rd }}$ main cause then use of red chili and inheritance are also two main consequences which are greatly involved in mouth cancer not as that of tobacco and alcohol but as much as sunlight. Sunlight being the last of all is also not neglect able and to some extent is involved in different types of mouth cancers. Patients with blood group A are more likely to develop oral cancer than any other blood group the reason for this is unknown but its finding in this survey is very low. Fig.2 also shows that the tobacco is the largest cause of developing oral cancer and its prevalence is very high than any other factor.

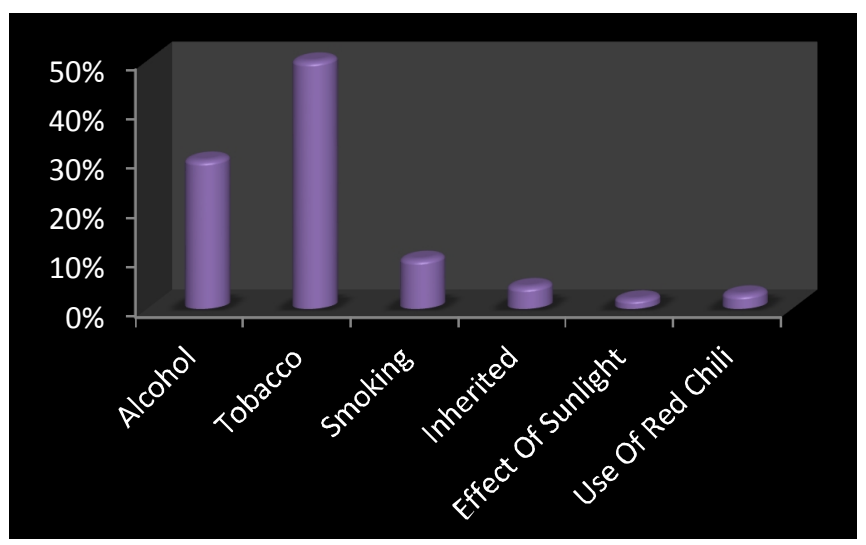

Figure 1: Different Consequences of Oral Cancer Showing Their Percentage of Occurrence

\section{DISSCUSSION}

Oral cancer is now one of the most spreading types of cancer. And to diminish this form of cancer it is very important to look out or find out those factors or consequences that are responsible for the development of this cancer. So for this purpose this study was conducted and consequences were found out.

Finding of consequences of oral cancer was such a difficult task because the methodology that was adopted for this study was very time consuming as well as efforts consuming but it lead to the very important findings.

On asking the patients about their history the most distressing situation was that the most of the patients were belonged to such areas where there is no preventive and careful managements were taken related to oral hygiene. And the height of carelessness was seen when some of the patients said that they even don't brush the tooth. Some patients were fair enough as they were educated ones and also very active towards oral hygiene but the reason for their cancer was 
inheritance. Also some patients' occupation was the reason for their disease as they work continuously in sunlight which is as said before is somewhat responsible for oral and many other types of cancers.

Some patients were found of using high amount of red chili in their meals and this deed then results in the development of their disease. Some patients were also affected due to the use of reused of dental instrument by their physicians.

The most common type found in patients was squamous cell carcinoma. And the most important reason for the development of oral cancer is the use of tobacco (mainly gutka, mava) which was found in most of the patients of about $50 \%$. Alcoholic patients were also found of about $30 \%$ then smoking $10 \%$. The combined effect of tobacco and alcohol is much greater than that of their sum means if a person take these two together, has a greater risk of developing oral cancer as compared to those 2 individuals who are taking any one of them.

In early development there is no cancer, may referred as pre cancerous lesion. So it is necessary to consult the doctor as soon as possible before this pre malignant lesion is transformed to the cancerous one. It can also be recognized by some symptoms in the oral cavity like fungating mass, erythroplakia, leukoplakia or some kind of disruption and erosion. These all are pre cancerous signs which later can be developed into the modified form.

\section{CONCLUSION}

This whole study concluded that the most occurring consequence of oral cancer is tobacco. Likewise many other factors are also involved like alcohol, smoking, inherited factors and use of red chili. And these all consequences are responsible for the prevalence of oral cancer. And this prevalence can be decrease by withdrawing of such agents that cause oral cancer like tobacco (gutka), smoking and alcohol in our society. And can also be diminished by avoiding the use of reused instruments by dentist during oral procedure and surgery or by using sterile instrument and also by maintaining oral hygiene. $z$

Also these hazardous agents that results in oral cancer should be banned by the government in order to stop the incidence of oral cancer especially in Karachi because Karachi is considered to be one of the largest city which use these agents. So it should be banned in Karachi on first hand.

Awareness programs should also be conducted specially in those areas where people are not aware of maintaining their oral hygiene in order to tell people about the facts and sign and symptoms of oral cancer and their immediate consultation to doctors so that cancerous condition should be avoided.

And above written measures can be taken to reduce the prevalence and risk of oral cancer in our society and to promote healthy and peaceful environment in our society.

\section{ACKNOWLEDGMENTS}

Authors would like to acknowledge Dr. Maria Ayub (our teacher and a hospital pharmacist) who helped us to get permission for the survey of different hospitals for the collection of data. Without this no survey would have been possible.

\section{REFERENCES}

1. Jatin $\mathrm{P}$ et al,Shah, Ziv Gil: current concepts in management of oral cancer-surgery. Journal of oral oncology. 2009 Vol. 45(4-5) :394-401 doi:10.1016/j.oraloncology.2008;05.017

2. Bartsch H et al, Nair U, Risch A, Rojas M, Wikman H, Alexandrov K: Genetic Polymorphism of CYP Genes, Alone or in Combination, as a Risk Modifier of Tobacco-related Cancers. Cancer Epidemiology Biomarkers \& Prevention 2000; 9:3-28.

3. Moiuddin Mlamgir et al, Qamar Jamal, N.A Jafery, Tallat Mirza. Pakistan Journal of Medical and Dentistry. 2013;Vol .2(2): 3-8.

4. Nair UJ et al, Nair J, Mathew B, Bartsch M. Glutathione Stransferase $\mathrm{M} 1$ and $\mathrm{T} 1$ null genotypes as risk factors for oral leukoplakia in ethnic Indian betel quid/tobacco chewers. Carcinogenesis 1999; 20(5):743-748.

5. Pakistan Medical Research Council. Collection of data of various types of tumors in Pakistan. PMRC Monograph 1977;1:58.

6. Zaidi SHM et al, Jafarey NA, and Askari A. Malignant tumors in Pakistan: a multicentre study 1977-80. Karachi: PMRC, JPMC., 1982:60p.

7. Dr. Brad W et al,Newlle DDS1 and Dr. Terry A. Oral cancer precancerous lesions : A cancer Journal For Clinicians.Day MD, FASC2, 23feb2009.CA.vol. 52. Issue 4: 195-215, july/august 2002

8. Y . k. Chen et al, H. C. Huang, L. M. Lin, and C. C. Lin. Primary oral squamous cell carcinoma for analysis of 703 cases in southern Taiwan .Oral Oncology. 1999;Vol. 35(2): 173-179.

9. Richard L et al. Ocuerrant, M.D, Margaret koset,. The New Engl Journal med. Vol. April 26, 2001;344(17).

10. A Villa et al, c Villa, s Abati. Oral cancer and oral erythroplakia; an update and implication for clinicians. Australian Dental Journal .2011;vol. 56: 253-256.

11. Christan Nordqvist, $9^{\text {th }}$ dec 2012. American Journal of epidermiology. Mouth cancer and causes.

12. Petersen PE. Oral cancer prevention and control the approach of the world health organization. Oral Oncol 2009; Vol. 45: 54-460.

13. Stephen M et al. Schwartz, Janet R. Daling and Margaret M. Madelein, Gregory C. Wipf, Joseh J. Carter, Er-Jia Mao, Shixuan Huang and Anna Marie Beckmann, James K. McDougall, Denise A. Gallaway. Oral cancer in relation to sexual history and evidence of Human Papillomavirus Infection. Vol. 90(21): 16261636.

14. Y. Niu et al, H. Yuan, M. Shen, H. Li, Y. Hu, and N. Chen. Journal of Craniofacial surgery. Association between cyclooxygenase-2 gene polymorphisms and head and neck squamous cell carcinoma risk. 2014;Vol. 25(2): 333-337.

15. Dong Li et al, Shu-hong Hao, Yan Sun, Chun-Mie Hu, Zhi-Hua ma, Zhi Ming Wang, Jie Liu, Hong Bo Liu, ming Ye, Yu Fie Zhang, Dong Sheng Yang, Guang Shi. Functional Polymorphisms in COX-2 gene are Correlated with the risk of Oral cancer. Journal of biomed Research International . 2015; Vol. 2015: 12.

16. H. H. H. W et al. Schmidt and U. Walter. "No at Work," Cell. 1994;Vol. 78(6): 919-925.

17. S. T et al. Connelly, M. Macabeo-ong, N. C. K. Jordan, and B. L. Schmidt.. Increased nitric oxide levels and iNOS over-expression in oral squamous cell carcinoma. Oral Oncology .2005; Vol. 41(3): 261-267.

18. Lan Yang et al, Youyuan Wang, Lvhua Guo, Liping Wang, Weiliang Chen, and Bin Shi. The expression and correlation of iNOS and P53 in Oral Squamous Cell Carcinoma. Biomed Research International Article. 2015.

19. Rene Leemans et al, Boudewijin J. M. Braakhuis \& Ruud H. Brakenhoff. C. The Molecular biology of head and neck cancer. Nature Reviews Cancer .2011;Vol. 11: 9-22

20. Raghu Dhanapal et al, K. Ranaganathan, Paturu kondaiah, R. Uma Devi, Elizabeth Joshua, and T. R. Saraswathi. High-risk human papillomavirus in archival tissues of oral pathosis and normal oral mucosa. Contemporary clinical dentistry .2015; Vol. 6(2): 148-152.

21. Smeuls J et al. Hooper, St John Crean, micheal A. O Lews, David A. Spratt, Willians G. Wade and Nelanie J. Wilson. Visible bacteria present within oral squamous cell carcinimas tisses. Journal of clinical microbiology. may 2006; Vol. 44(5): 17191725. 
22. Flavio Juliana Pimenta et al, Gabriela Tavares Cordeiro, Luiz Gustavo Garcia Santos Pimenta, Michelle Beatriz Viana, Joyce lopes, Marcus Vinicius Gomez, C. Marcelo Aldaz, Luiz De Marco, Ricardo Santiago Gomez, Molecular alteration in the tumor suppressor gene. WWOX in oral leukoplakias.. Journal of oral oncology. 2008; Vol. 44(8): 753-758.

23. Guanghui Zhu et al, Yuan He, Shaofang Yang, Beimin chen, Min Zhou, And Xin-Jian Xu, 2015. BioMed Research International. Identification of Gene And MicroRNA Signature for Oral Cancer Developed from Oral Leukoplakia. Vol. 2015.

24. Radhakrishna Pillai PhD et al, Prabha Balaram PhD, and Kanna Sankara Reddiar. Pathogenesis of oral sub mcous fibrosis. Relationship to risk factors associated with oral cancer. 2011; Vol. 69(8): 2011-2020.

25. Lissowska et al, J; pilarskai, P; samolczyk wanyura, D; piekarczyk, J; bardin-mikcllajczak' A; zatonski, W; herrero, R; munoz, N; franceshi, s. European Journal of Oral Cancer Prevention. 2013; Vol. 12(1): 25-33.
26. Madhuri S et al, Govind Pandey*, Rita Bhandari and A.B. Srivastava. Contamination of polychlorinated biphenyls in fishes leading to toxicity in fish consuming population Int Res $\mathbf{J}$ Pharm 2012;3(10):7-9

27. Maria Ayub*et al, Kainat Firdous, Huma Rizwan, Safeena Siddique, Sumiya Khan, Ushna Maryam.Awareness status of breast cancer in pakistan society among affected women with breast cancer and control men and women: a cross-sectional study, Journal of Chemical, Biological and Physical Sciences, 2015, Vol. 5, No. 3

\section{Cite this article as:}

Maria Ayub, Mehreen fayyaz, Sumra arshad, Bushra riaz khan, Uzma bano, Falak khalil. Oral cancer prevalence \& finding of alarming consequences at oncology ward of public health care sector. Int. Res. J. Pharm. 2015; 6(9):623-626 http://dx.doi.org/10.7897/2230$\underline{8407.069121}$

\section{Source of support: Nil, Conflict of interest: None Declared}

Disclaimer: IRJP is solely owned by Moksha Publishing House - A non-profit publishing house, dedicated to publish quality research, while every effort has been taken to verify the accuracy of the content published in our Journal. IRJP cannot accept any responsibility or liability for the site content and articles published. The views expressed in articles by our contributing authors are not necessarily those of IRJP editor or editorial board members. 